\title{
EL USO DE TÉCNICAS DE CONTABILIDAD GERENCIAL EN LA TOMA DE DECISIONES DE LAS PYMES DE LA GRAN AREA METROPOLITANA DE COSTA RICA
}

\author{
LUIS VALVERDE RAMÍREZ \\ Escuela de Ciencias de la Administración \\ Universidad Estatal a Distancia, Costa Rica \\ Ivalverde9@gmail.com
}

\begin{abstract}
RESUMEN
Las Pequeñas y Medianas Empresas (Pymes) de Costa Rica juegan un papel fundamental en la generación de empleo y el desarrollo del país. Aunque constantemente deben tomar decisiones relacionadas con precios, costos, inversiones y nuevos negocios, con frecuencia desconocen las técnicas de Contabilidad Gerencial disponibles para esos fines. La mayoría de estas técnicas son prácticas, sencillas y económicas. A pesar de esto, su uso por parte de las Pymes es muy escaso. Con frecuencia, decisiones que se pueden tomar de manera técnica son resueltas con base en el instinto, intuición o costumbre. A la vez, la poca estructura interna, escasez de recursos, presiones por sobrevivir y escasa capacitación, inciden en el bajo nivel de aplicación de las técnicas de Contabilidad Gerencial. Existe una oportunidad importante para que las universidades colaboren con las Pymes ofreciéndoles capacitación y herramientas sencillas que les permitan tomar mejores decisiones, mediante el uso extensivo de las técnicas de Contabilidad Gerencial.
\end{abstract}

\section{PALABRAS CLAVE: PYMES, CONTABILIDAD GERENCIAL, TOMA DE DECISIONES.}

\section{ABSTRACT}

Costa Rica's SMEs play a key role in the generation of employment and the development of the country. Although they must constantly make decisions concerning prices, costs, investment and new businesses, they do not often know management accounting techniques available for these purposes. Most of these techniques are practical, simple and economical. However, their use by SMEs is very occasional. Frequently, decisions may be resolved technically but are actually made based on instinct, intuition, or custom. At the same time, the lack of an internal structure, shortage of resources, pressure to survive and little training have an impact on the low level of implementation of management accounting techniques. There is an important opportunity for universities to collaborate with SMEs by providing them with training and simple tools to enable them to make better decisions through the extensive use of management accounting techniques.

KEYWORDS: SMALL BUSINESS, MANAGEMENT ACCOUNTING, DECISION MAKING.

\section{ANTECEDENTES}

La Cátedra de Contabilidad Superior de la Escuela de Ciencias de la Administración de la UNED en Costa Rica desarrolló el proyecto de Investigación denominado "El Uso de Técnicas de Contabilidad Gerencial en la Toma de Decisiones de las Pymes de la Gran Área Metropolitana (GAM)." Este artículo proviene de dicha investigación.

Existe una amplia gama de estas técnicas que fueron diseñadas para apoyar la toma de decisiones en las empresas, instituciones y Organizaciones No Gubernamentales (ONG's). A nivel internacional, las técnicas de Contabilidad Gerencial (análisis de precios, análisis de costos relevantes, presupuestos, punto de equilibrio, precios de trans- 
ferencia, costeo basado en actividades, costeo por objetivos, evaluación del desempeño, evaluación de outsourcing, análisis de sustitución de equipos, análisis de contratos especiales, entre otros) son cada vez más utilizadas, al igual que sucede en compañías locales grandes y en multinacionales. Por su parte, las Pymes emplean estás técnicas fundamentales en menor escala, a pesar de ser muy relevantes para tomar decisiones acertadas en situaciones de negocio frecuentes, tanto operativas como estratégicas. Por lo tanto, es posible que muchas decisiones se tomen bajo criterios subjetivos o meramente intuitivos, a pesar de que existen herramientas técnicas para analizar las situaciones e identificar las decisiones adecuadas. Estas herramientas son en su mayoría relativamente sencillas, accesibles y de bajo costo.

Dada la importancia para su éxito, es conveniente establecer el grado de conocimiento que tienen las Pymes de las técnicas de Contabilidad Gerencial (también Ilamada contabilidad administrativa o de gestión), cuáles de ellas utilizan y en qué medida, cuáles no son utilizadas y por qué razones. Asimismo, se debe conocer el grado de formación de los diferentes responsables de tomar las decisiones relacionadas con las técnicas de Contabilidad Gerencial.

En este artículo se pretende identificar el grado de conocimiento y uso de las técnicas de Contabilidad Gerencial en una muestra de Pymes de la GAM de Costa Rica. Considerando además identificar los responsables de toma de decisiones en situaciones relacionadas con técnicas de Contabilidad Gerencial, establecer el grado de conocimiento que tienen los tomadores de decisiones de las Pymes de las técnicas de Contabilidad Gerencial, establecer en qué medida, los responsables de la toma de decisiones de las Pymes aplican las técnicas de Contabilidad Gerencial en sus problemas cotidianos e identificar las áreas de la Contabilidad Gerencial en que las
Pymes requieren mayor formación, asesoría o acompañamiento.

\section{Características de las Pymes de Costa Rica}

Las Pymes se definieron de conformidad con los criterios del Observatorio de Mipymes de la UNED (Observatorio de Mipymes, 2008), que establecen que la pequeña empresa tiene entre 6 y 30 trabajadores y la mediana entre 31 y 100.

Las estadísticas de la Caja Costarricense de Seguro Social, a diciembre del año 2009, muestran las siguientes características relevantes de las Pymes de Costa Rica:

1. Las Pymes generan el $38 \%$ del empleo nacional. El 21\% corresponde a pequeñas empresas y el $17 \%$ a medianas.

2. Las Pymes contaban con aproximadamente 289.000 trabajadores a diciembre de 2009, de los cuáles 160,000 laboran en pequeñas empresas y 129.000 en medianas. Estas cifras corresponden, respectivamente, al 20.7 $\%$ y $16.7 \%$ del empleo nacional.

3. Las Pymes concentran su actividad en el comercio y servicios (más del $50 \%$ ) y en segundo lugar en la industria.

4. La cantidad de Pymes ha aumentado considerablemente en los últimos años.

La UNED ha realizado un importante estudio (Observatorio de Mipymes, 2008) sobre este sector de la economía. Aunque incluyó también a las microempresas (que no se consideran en este artículo), es importante conocer y utilizar la valiosa información obtenida. Las conclusiones señalan que: la importancia de las Mipymes es notable por la cantidad de empresas, el empleo que generan y las exportaciones que realizan; la cantidad de Mipymes ha aumentado en los últimos seis años; la mayoría de las empresas en Costa Rica por tamaño son microempresas y por 
actividad son de servicios; la semiformalidad es alta; la tasa de rotación de las Mipymes es elevada y la tendencia a aliarse para lograr objetivos comerciales es baja, sin embargo, se duplica en las empresas exportadoras.

\section{MARCO TEÓRICO}

Los principales desarrollos de las técnicas de contabilidad gerencial se dieron en la segunda parte del siglo XX (Neuner, 2001; Horngren, 2007; Garrison, 2007) y continúan en el presente. Las técnicas de contabilidad gerencial combinan conocimientos provenientes de la contabilidad financiera, contabilidad de costos, finanzas corporativas, microeconomía, matemáticas, investigación de operaciones y estadística. Fundamentalmente, tratan sobre la evaluación económica de alternativas relacionadas con precios, costos, operaciones e inversiones. Usualmente el análisis se basa en la valoración de las diferencias entre esas alternativas de decisión. Este proceso se denomina a veces análisis de costos relevantes, análisis marginal, análisis incremental, análisis diferencial, entre otros. Conforme avanza el conocimiento en este campo, se han observado tendencias relevantes tales como:

1. Mayor énfasis en el valor práctico de las técnicas de análisis de costos en la toma de decisiones.

2. Mayor aplicación de las técnicas de contabilidad gerencial en todo tipo de organizaciones, públicas y privadas, grandes o pequeñas.

3. Mayor incorporación de cursos sobre técnicas de contabilidad gerencial en los programas de negocios, contaduría y economía de la empresa.

4. Mayor uso de herramientas estadísticas en la aplicación de las técnicas de contabilidad gerencial.
5. Mayor uso de herramientas informáticas (hojas de cálculo y programas especializados) en la toma de decisiones basada en técnicas de contabilidad gerencial.

6. Mayor convergencia e integración en herramientas específicas, de las técnicas provenientes de la contabilidad de costos, finanzas corporativas, microeconomía, matemáticas, investigaciones de operaciones y estadística.

7. Mayor aplicación de las técnicas de contabilidad gerencial a operaciones internacionales.

8. Mayor uso de las técnicas de contabilidad gerencial en la toma de decisiones estratégicas.

9. Mayor interrelación entre las técnicas clásicas de contabilidad gerencial y herramientas tales como control de calidad, six sigma, cuadro de mando integral, teoría de las restricciones y análisis de la productividad.

\section{METODOLOGÍA}

La metodología utilizada incluye los siguientes puntos:

1. Revisión del marco normativo de las Pymes de Costa Rica.

2. Revisión de literatura sobre técnicas de contabilidad gerencial.

3. Revisión de diversos estudios sobre Pymes en Latinoamérica.

4. Revisión de información cualitativa y cuantitativa de Pymes de Costa Rica.

5. Resumen de los principales resultados de la investigación "El Uso de Técnicas de Contabilidad Gerencial en la Toma de Decisiones de las Pymes de la Gran Área Metropolitana," realizada por el suscrito para UNED. 
6. Realización de entrevistas a los gerentes de Pymes investigadas, a fin de ofrecer un espacio abierto de diálogo sobre el uso de técnicas de contabilidad gerencial.

\section{RESULTADOS}

Los resultados muestran el perfil de la Pymes estudiadas, los responsables de tomar decisiones en materia de precios, costos, inversión y nuevos negocios, su escolaridad, el nivel de conocimiento con que cuentan respecto a las principales técnicas de contabilidad gerencial y la frecuencia con que las aplican en las empresas en que laboran.

\section{Perfil de las Pymes de la muestra}

Se consultaron 30 Pymes de la Gran Área Metropolitana de Costa Rica, a fin de perfilar sus necesidades en materia contabilidad gerencial. Fueron elegidas mediante muestreo por conveniencia y con reemplazo. Las Pymes seleccionadas muestran el siguiente perfil:

1. Clasificación Industrial: tres agroindustriales, ocho comerciales, diez industriales y nueve de servicios.

2. Empleados por Pymes: el promedio es de 14 para Pymes agroindustriales, 41 para comerciales, 40 para industriales y 28 para empresas de servicios. El promedio general es de 33 .
3. Antigüedad de las Pymes: tres de 0 a 5 años, seis de 6 a 10 años y 21 con más de 10 años.

4. Pymes que venden al Sector Público: 16 venden al Sector Público y 14 no lo hacen.

5. Pymes que exportan: 14 exportan bienes o servicios, en tanto que 16 no lo hacen.

6. Contador de planta o externo: 21 cuentan con Contador de planta y 9 con Contador externo.

Responsabilidad por decisiones de precios, costos, inversión y nuevos negocios

Los cargos que en las Pymes se responsabilizan por las decisiones de precios, costos, inversiones y nuevos negocios se presentan en la Tabla 1.

Escolaridad de quienes toman

decisiones de precios, costos,

inversión y nuevos negocios

En la tabla 2 se muestra la escolaridad de los responsables de tomar los principales tipos de decisiones en la Pymes. En algunos casos, estas decisiones son tomadas por un cuerpo colegiado (Junta Directiva), por lo que la escolaridad puede variar de un miembro a otro.

TABLA 1

Responsabilidad por la toma de desiciones en las Pymes

\begin{tabular}{|l|c|c|c|c|}
\hline \multirow{2}{*}{ Cargo } & \multicolumn{4}{|c|}{ Tipo de desición } \\
\hline Gerente General & $60,0 \%$ & $53,3 \%$ & $53,3 \%$ & Costos \\
\hline Gerente de Ventas & $23,4 \%$ & $0,0 \%$ & $0,0 \%$ & $0,0 \%$ \\
\hline Junta Directiva & $10,0 \%$ & $6,7 \%$ & $30,0 \%$ & $30,0 \%$ \\
\hline Gerente Financiero & $3,3 \%$ & $30,0 \%$ & $10,0 \%$ & $10,0 \%$ \\
\hline Contador & $0,0 \%$ & $10,0 \%$ & $0,0 \%$ & $0,0 \%$ \\
\hline Propietario & $3,3 \%$ & $0,0 \%$ & $6,7 \%$ & $6,7 \%$ \\
\hline
\end{tabular}

Fuente: elaboración propia. 
TABLA 2

Escolaridad de responsables de toma de desiciones en las Pymes

\begin{tabular}{|l|c|c|c|c|}
\hline \multirow{2}{*}{ Escolaridad } & \multicolumn{4}{|c|}{ Tipo de desición } \\
\hline Maestría & $20,0 \%$ & $23,3 \%$ & $23,3 \%$ & $23,3 \%$ \\
\hline Licenciatura & $23,3 \%$ & $26,7 \%$ & $26,7 \%$ & $16,7 \%$ \\
\hline Bachillerato universitario & $30,0 \%$ & $30,0 \%$ & $20,0 \%$ & $23,3 \%$ \\
\hline Parauniversitaria & $10,0 \%$ & $13,3 \%$ & $10,0 \%$ & $10,0 \%$ \\
\hline Bachiller secundaria & $6,7 \%$ & $0,0 \%$ & $0,0 \%$ & $6,7 \%$ \\
\hline Junta Directiva & $10,0 \%$ & $6,7 \%$ & $20,0 \%$ & $20,0 \%$ \\
\hline
\end{tabular}

Fuente: elaboración propia.

\section{Conocimiento de las técnicas de contabilidad gerencial del grupo 1}

Las técnicas de contabilidad gerencial se dividieron en dos grupos, considerando la complejidad relativa, extensión de uso y novedad. Las técnicas de contabilidad gerencial del grupo 1 son, en general, más simples, conocidas y antiguas. La lista de técnicas del grupo 1, ordenadas de más conocidas a menos conocidas, es la siguiente:

1. Análisis del punto de equilibrio.

2. Elaboración y control presupuestos.

3. Análisis económico de descuentos y promociones.

4. Análisis de contratos especiales a precios reducidos.

5. Mantener, eliminar o agregar segmentos de negocios.

6. Análisis de costos relevantes para fijación de precios.

7. Evaluación de centros de costos y utilidad.

8. Análisis de costeo absorbente y variable.

9. Análisis económico de fabricar o comprar.

10. Análisis de la conveniencia de venta en el punto actual o de procesamiento adicional.
El Gráfico 1 señala qué porcentaje de las Pymes de la muestra manifiestan conocer las técnicas de contabilidad gerencial del grupo 1.

GRÁFICO 1

Resumen conocimiento técnicas de Contabilidad de Gerencia en Pymes (Grupo 1)

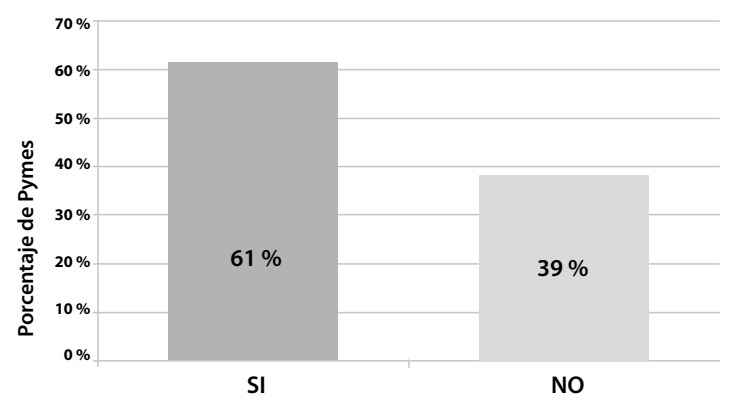

Fuente: elaboración propia.

\section{Conocimiento de las técnicas de Contabilidad Gerencial del Grupo 2}

La lista de técnicas de contabilidad gerencial del grupo 2, ordenadas de más conocidas a menos conocidas, es la siguiente:

1. Análisis económico de alquilar o comprar.

2. Análisis económico de la conveniencia de contratos tipo outsourcing.

3. Análisis económico de reemplazo equipos.

4. Costos estándar y análisis de variaciones de costos. 
5. Análisis económico de la productividad parcial y total.

6. Análisis de mezcla óptima de insumos.

7. Análisis económico de la cadena valor.

8. Análisis económico de productos conjuntos.

9. Costeo basado en actividades.

10. Análisis del cuadro de mando integral.

11. Análisis de precios transferencia.

12. Análisis estratégico de costos.

13. Costeo por objetivos.

El Gráfico 2 señala qué porcentaje representan las Pymes de la muestra que manifiestan conocer las técnicas de contabilidad gerencial del Grupo 2.

\section{GRÁFICO 2}

Resumen conocimiento técnicas de Contabilidad de Gerencia en Pymes (Grupo 2)

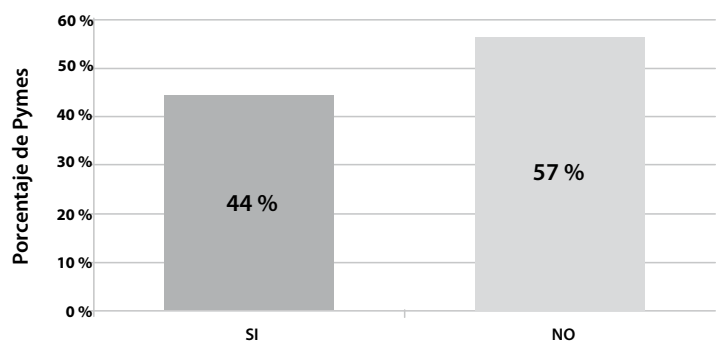

Fuente: elaboración propia.

\section{Resumen del conocimiento de las técnicas de} Contabilidad Gerencial de los Grupos 1 y 2

El Gráfico 3 resume la proporción de Pymes que manifiestan conocer las técnicas de Contabilidad Gerencial de los Grupos 1 y 2.
GRÁFICO 3

Resumen conocimiento técnicas de Contabilidad de Gerencia en Pymes (Grupo 1 y 2)

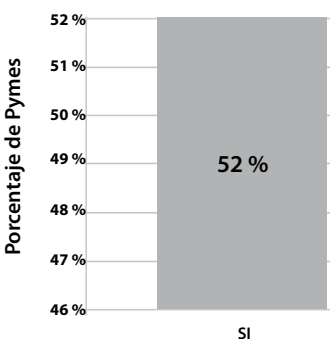

SI

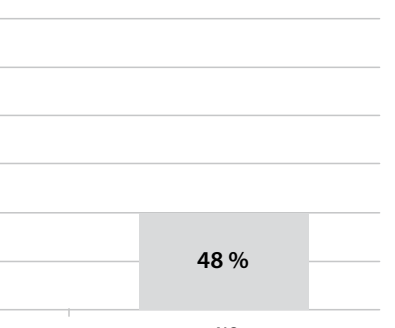

NO
Fuente: elaboración propia.

Aplicación de las técnicas de Contabilidad Gerencial del Grupo 1

El Gráfico 4 muestra en qué medida las Pymes estudiadas manifiestan que aplican las técnicas de contabilidad gerencial del Grupo 1 .La indicación de que una técnica es aplicada "siempre", significa que se utiliza cotidianamente, la mayor parte del año. Si se usa "bastante", indica que se aplica entre 7 y 12 veces al año; "regular" indica que se aplica entre 3 y 6 veces por año y "poco" indica que la usa menos de una a tres veces por año.

GRÁFICO 4

Resumen aplicación de técnicas de Contabilidad de Gerencia en Pymes (Grupo 1)

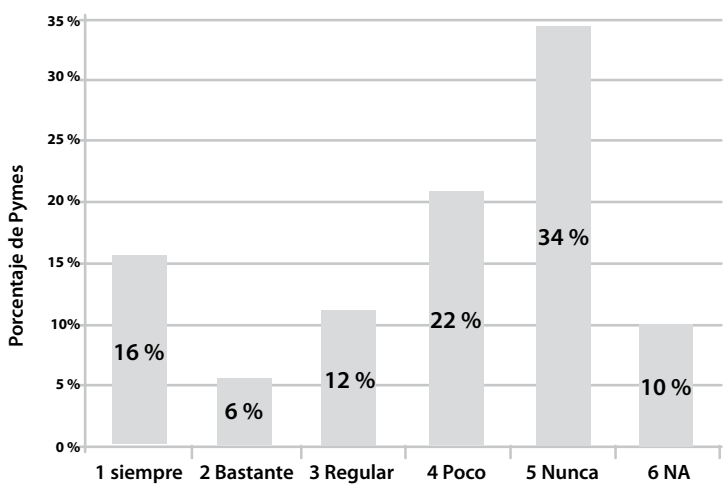

Fuente: elaboración propia. 
Los datos detallados muestran que las técnicas de presupuesto, evaluación de centros de costos y utilidades y análisis de punto de equilibrio son las más utilizadas. Se trata de técnicas maduras, con amplia tradición.

\section{Aplicación de las técnicas} de Contabilidad Gerencial del Grupo 2

El Gráfico 5 indica en qué medida las Pymes de la muestra manifiestan que aplican las técnicas de contabilidad gerencial del Grupo 2.

GRÁFICO 5

Resumen aplicación de técnicas de Contabilidad de Gerencia en Pymes (Grupo 2)

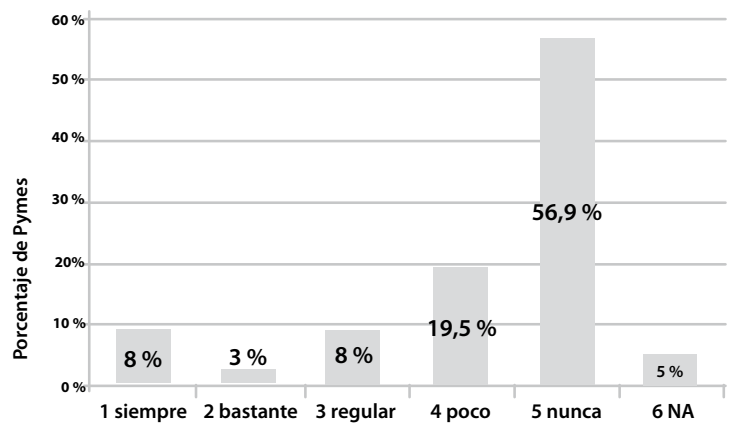

Fuente: elaboración propia.

Las técnicas del Grupo 2 se aplican en menor medida que las técnicas del Grupo 1. En efecto, el 34\% de las Pymes estudiadas indicó que utiliza las técnicas del Grupo 1 con una frecuencia entre "regular" $y$ "siempre", en tanto que, para ese mismo rango, utiliza las técnicas del Grupo 2 tan solo con una frecuencia de 19\%.

Resumen de la aplicación de las técnicas de Contabilidad Gerencial de los Grupos 1 y 2

El Gráfico 7 muestra la medida en que, en promedio, los responsables de las Pymes manifiestan que aplican las técnicas de contabilidad gerencial de los Grupos 1 y 2.
GRÁFICO 6

Resumen aplicación de técnicas de Contabilidad de Gerencia en Pymes (Grupo 1 y 2)

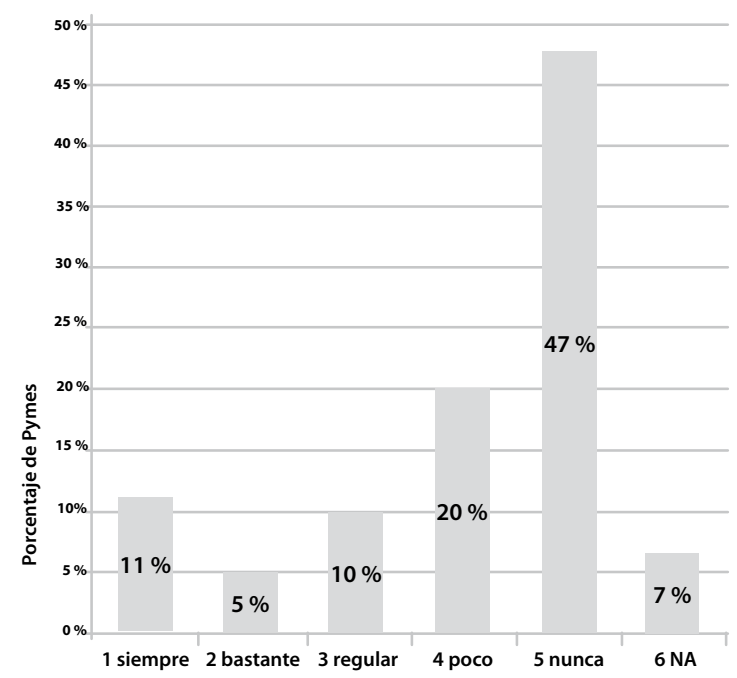

Fuente: elaboración propia.

Puede observarse que el grado de aplicación de las técnicas de Contabilidad Gerencial en general es muy bajo. En tanto que las Pymes manifestaron conocer en promedio el 52\% de las técnicas de los Grupos 1 y 2, solo las aplican con cierta frecuencia (de "regular" a "siempre") en el $26 \%$ de los casos.

\section{Capacitación recibida en los últimos tres años}

El Gráfico 7 indica cuáles de las 30 empresas de la muestra manifestaron que sus empleados recibieron capacitación en algunos temas técnicos de gran relevancia para la toma de decisiones de las Pymes. Esto temas son los siguientes: uso de hoja electrónica (MS-Excel), fijación de precios, análisis de costos, outsourcing, técnicas de presupuestos y cuadro de mando integral. 
GRÁFICO 7

Pymes cuyo personal recibió capacitación en temas selectos durante últimos 3 años

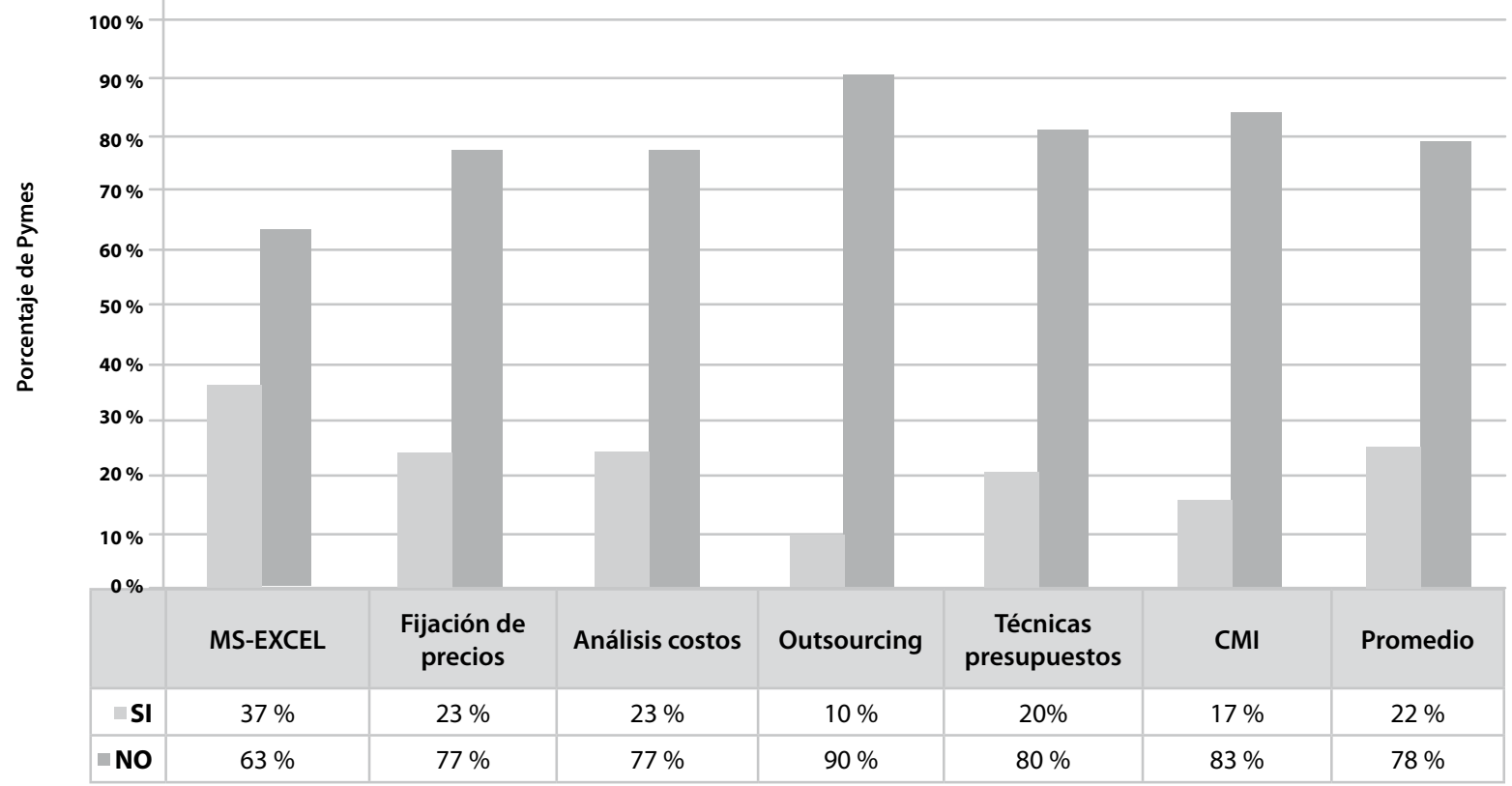

Se nota que, no obstante la importancia de la capacitación para el éxito de las Pymes, en promedio solo cerca de la quinta parte ha capacitado a personal de toma de decisiones en los temas citados.

\section{ANÁLISIS DE RESULTADOS Y ENTREVISTAS}

Seguidamente se resumen los resultados de la investigación realizada y entrevistas practicadas.

\section{Resultados}

La mayoría de las Pymes analizadas son de San José y se dedican a la industria, servicios y comercio y tienen entre 6 y 98 empleados, con un promedio de 33. En la muestra predominan las Pymes con más de 10 años y son escasas las que cuentan con menos de 5. Cerca de la mitad de las Pymes analizadas venden servicios al Sec- tor Público y/o exportan. El 70\% dispone de un Contador de planta.

Las decisiones de precios las toma, en la mayoría de los casos, el Gerente General y, en segundo lugar, el Gerente de Ventas. Las que se refieren a costos las toma nuevamente el Gerente General $y$, en segunda instancia, el Gerente Financiero.

Las decisiones de inversión y de emprender negocios son asumidas por la Gerencia General y, en segundo término, por la Junta Directiva.

El nivel de escolaridad predominante en los tomadores de decisiones es universitario (bachillerato, licenciatura y maestría). En muchos casos, existe clara conciencia de la necesidad de tomar decisiones fundamentadas en las técnicas disponibles.

Las técnicas del Grupo 1 son conocidas en promedio por el $61 \%$ de las Pymes estudiadas. Las técnicas más conocidas son 1) el análisis del 
punto de equilibrio, 2) la elaboración y control de presupuestos y 3) el análisis de descuentos y promociones.

Las técnicas del Grupo 2 son conocidas en promedio por el $44 \%$ de las Pymes de la muestra. Dentro de estas, las tres más conocidas son a) Análisis económico de alquilar o comprar, b) análisis económico del outsourcing, tanto activo (la empresa contrata) como pasivo (la empresa es contratada) y 3) el análisis económico de reemplazo de equipos.

En general, las Pymes analizadas manifiestan conocer en promedio cerca de la mitad de las técnicas de Contabilidad Gerencial que les fueron consultadas (Grupos 1 y 2). Las técnicas menos conocidas corresponden a 1) costeo por objetivos, 2) análisis estratégico de costos y 3) análisis de precios de transferencia. En general, el conocimiento de estas técnicas es claramente insuficiente.

Las técnicas del Grupo 1 se aplican con una frecuencia de "regular" o mayor en cerca de un tercio de los casos. La técnica más aplicada en este grupo es la de elaboración y control de presupuestos y la menos utilizada es la de análisis económico de fabricar o comprar.

En cuanto al Grupo 2, las técnicas se aplican con una frecuencia de "regular" o mayor en aproximadamente una de cada seis empresas de la muestra. La técnica del grupo 2 más utilizada es la de costos estándar y análisis de variaciones de costos.

En general, las Pymes analizadas indican que aplican, en promedio, cerca de la cuarta parte de las técnicas de Contabilidad Gerencial que les fueron consultadas (Grupos 1 y 2). Las técnicas de menor utilización son el costeo por objetivos y el análisis económico de reemplazo de equipos. Este nivel tan escaso de aplicación de las técnicas disponibles limita las posibilidades de supervivencia y crecimiento de las Pymes.

Los responsables de las Pymes reconocen la importancia de la capacitación para su supervivencia, crecimiento y éxito futuro. No obstante, invierten relativamente poco tiempo y dinero en esta actividad. En promedio, cerca de una de cada cinco ha capacitado a alguien de su personal en temas relevantes de Contabilidad Gerencial. Los temas consultados incluyen la hoja de cálculo MS-Excel, técnicas de fijación de precios, análisis de costos para toma de decisiones, análisis del outsourcing, técnicas de presupuestos y cuadro de mando integral.

La poca capacitación recibida, el alto grado de desconocimiento de las técnicas de Contabilidad Gerencial y su bajo nivel de aplicación, representan una importante oportunidad de mejora para las Pymes.

\section{Entrevistas}

Durante las entrevistas y conversaciones abiertas sostenidas con los empresarios al aplicar el cuestionario, se recibieron comentarios de interés como los siguientes:

Los contadores de planta generalmente asumen otras labores administrativas que presionan su jornada normal, lo que obstaculiza realizar, con mayor frecuencia y rigor, análisis técnicos en apoyo de la toma de decisiones.

La estructura organizativa, en la mayoría de los casos, es muy sencilla y limitada, lo que hace que el Gerente General y algunos de sus principales subordinados asuman diversas tareas necesarias pero de menor complejidad. Esta práctica tiende a diluir los esfuerzos y prioridades.

Evitan contratar personal muy calificado, para no recargar la planilla con su costo asociado. Esto 
contribuye a que se recarguen las funciones entre el escaso personal disponible y a que las decisiones de cierta complejidad con frecuencia no reciban el análisis que requieren.

Una cantidad importante de los entrevistados no conoce herramientas especializadas de hoja de cálculo que podrían ayudarle a tomar decisiones de manera más técnica.

Aunque se reconoce la importancia de la capacitación, otras prioridades tienden a absorber la mayor parte del tiempo y los recursos financieros de las Pymes.

Algunos responsables de Pymes reconocen haber estudiado en algún momento una técnica determinada, pero la han olvidado, o no la dominan suficientemente como para utilizarla con confianza, o la entienden en teoría pero nunca tuvieron la experiencia de aplicarla.

Una de las principales observaciones planteadas por los responsables de las Pymes, es que sus empresas han sido muy afectadas por la crisis económica, por lo que están concentrados fundamentalmente en la sobrevivencia y el día a día, lo que no deja mucho espacio para el esfuerzo que requiere fortalecer la gestión técnica.

Se da una paradoja en el sentido de que, al no conocer algunas técnicas, estas no se aplican, a pesar de que potencialmente pueden contribuir de manera significativa a resolver los problemas que más les preocupan.

Algunos gerentes aún consideran que muchas de estas técnicas son más apropiadas para las grandes empresas o las multinacionales y, por lo tanto, poco accesibles a las Pymes.

La mayoría de los gerentes manifestó estar dispuesto a participar en programas o eventos que les permitan mejorar su nivel de conocimiento y utilización de las técnicas de control gerencial.

\section{CONCLUSIONES}

Las Pymes desempeñan un papel clave en la economía nacional, especialmente por la generación de empleo en las áreas de comercio y servicio.

Las Pymes están sometidas a importantes presiones competitivas, no solo en el mercado privado local, sino también como proveedores del Sector Público y exportadores.

La toma de decisiones en materia de precios, costos, inversiones y negocios nuevos tiende a estar centralizada en el Gerente General, con participación del Gerente de Ventas en decisiones sobre precios y del Gerente Financiero en decisiones sobre costos.

La escolaridad de los gerentes de las Pymes de la muestra es relativamente alta, predominando los niveles universitarios. No obstante, un alto porcentaje de los entrevistados desconoce gran parte de las técnicas de contabilidad gerencial.

Cerca de la cuarta parte de las Pymes aplica con frecuencia una parte importante de las técnicas de contabilidad gerencial. Del $75 \%$ restante, dos tercios desconocen la mayoría de las técnicas y un tercio indica que las conoce pero no las aplica.

Durante los últimos tres años, solo cerca de la quinta parte de las Pymes han recibido capacitación en temas críticos como MS-Excel, fijación de precios, análisis de costos, análisis de outsourcing, técnicas de presupuestos y cuadro de mando integral.

En entrevistas personales, una parte importante de los gerentes que manifestaron conocer determinada técnica y no aplicarla, indicaron que se debía a: a) falta de tiempo, b) concentración en sobrevivir a la crisis económica, c) cuentan con poca estructura interna, d) consideran que estás técnicas son más apropiadas para grandes 
empresas o multinacionales, e) aunque estudiaron en la universidad algunas de las técnicas citadas, nunca tuvieron experiencia en su aplicación o las han olvidado, f) se acostumbraron a decidir por "olfato" y g) están iniciando el proceso de tecnificar las decisiones.

Algunos gerentes que no conocen las técnicas indicaron que a) los programas cursados en negocios no incluían temas de costos, b) su profesión no es la administración o c) no son profesionales universitarios.

El desconocimiento, sub aplicación o no aplicación de las técnicas de control gerencial representa un importante costo de oportunidad para las Pymes, en términos de las oportunidades de creación de valor desaprovechadas.

La mayoría de los participantes manifestó gran interés en recibir apoyo de la UNED vía capacitación, seminarios, charlas, conferencias, envío de artículos, estudios e investigaciones, así como en el desarrollo de opciones virtuales y locales de capacitación sobre las técnicas mencionadas en el estudio.

En general los entrevistados reconocen que, debido a las fuertes presiones competitivas que enfrentan, es urgente fortalecer el grado de conocimiento de las técnicas de contabilidad gerencial, así como aprovechar las ventajas que traería su aplicación en las Pymes.

\section{RECOMENDACIONES}

Estructurar acciones de capacitación que permitan fortalecer el conocimiento de las técnicas de contabilidad gerencial en las Pymes, incluyendo opciones tales como seminarios, charlas, foros, conferencias, talleres, cursos formales y en línea.
Programar eventos en zonas rurales para capacitar en técnicas de contabilidad gerencial, simultáneamente, a varias Pymes de la misma región.

Promover el uso amplio de las técnicas de contabilidad gerencial en las Pymes mediante el diseño de instructivos, guías e instrumentos (modelos de hoja de cálculo) que faciliten su comprensión y aplicación.

Disponer un espacio en la página Web del Observatorio de Mipymes de la UNED y un enlace en la página Web del Ministerio de Economía, Industria y Comercio de Costa Rica, para que los responsables de la toma de decisiones en estas empresas puedan acceder a una "caja de herramientas" de contabilidad gerencial diseñada para Pymes.

Realizar convenios con agencias extranjeras y organismos internacionales especializados en Pymes, para que permitan divulgar sus materiales de alta calidad relacionados con la contabilidad gerencial para Pymes.

Elaborar y publicar un libro de contabilidad gerencial enfocado en las necesidades de las Pymes latinoamericanas, que permita presentar los materiales técnicos con sencillez, adaptados a la escala de estas empresas, con modelos simplificados de aplicación directa e inmediata y con consejos prácticos para interpretar adecuadamente los resultados y prevenir errores.

En resumen, se recomienda contribuir desde la UNED a fortalecer la competitividad, eficiencia y eficacia de las Pymes, mediante el diseño e implementación de un programa de extensión de la Escuela de Ciencias de la Administración, que contribuya a ampliar y fortalecer su nivel de conocimiento y grado de aplicación de las técnicas de contabilidad gerencial. 
RNA Revista Nacional de Administración

\section{REFERENCIAS}

Garrison, R. (2007). Contabilidad Administrativa. México: McGraw-Hill.

Horngren, C. (2007). Contabilidad de Costos. México: Pearson.

Caja Costarricense de Seguro Social. (2010). Anuario Estadístico 2009. Costa Rica: CCSS.

Neuner, J. (2001). Contabilidad de Costos. México: Limusa.

Observatorio de Mipymes. (2008). Hacia el estado de las Mipymes: Primer diagnóstico nacional de Mipymes. Costa Rica: UNED.

Recibido: 04-07-2011

Aceptado: 03-10-2011 